\title{
Trends in incidence of primary brain tumors in the United States, 1985-1994 ${ }^{1}$
}

\author{
Patti J. Jukich, Bridget J. McCarthy, ${ }^{2}$ Tanya S. Surawicz, Sally Freels, \\ and Faith G. Davis
}

Division of Epidemiology and Biostatistics, School of Public Health, University of Illinois at Chicago,
Chicago, IL 60612 (P.J.J., B.J.M., T.S.S., S.F., F.G.D.); and Central Brain Tumor Registry of the United States,
Chicago, IL 60632 (P.J.J., B.J.M., T.S.S., S.F., F.G.D.)

Brain tumor incidence has increased over the last 20 years in all age groups, both overall and for specific histologies. Reasons attributed to these increases include increase in lymphoma due to HIV/AIDS, introduction of computed tomography/magnetic resonance imaging, and changes in coding/classification. The purpose of this study was to describe overall and histologic-specific incidence trends in a population-based series of primary benign and malignant brain tumors. Data from the Central Brain Tumor Registry of the United States from 1985 through 1994 were used to determine incidence trends in the broad age groups 0-19, 20-64, and $\geq 65$ years, both overall and for selected histologies. Poisson regression was used to express trends as average annual percentage change. Overall, incidence increased modestly (annual percentage change $0.9 \%, 95 \%$ confidence interval, $0.4,1.4)$. When lymphomas were excluded, this result was not statistically significant (annual percentage change $0.5 \%$, 95\% confidence interval, $-0.1,1.1$ ). Specific histologies that were increasing were lymphomas in individuals aged 20 to 64 years and in males aged 65 years or older, ependymo-

Received 5 October 2000, accepted 16 February 2001.

${ }^{1}$ This work was conducted under contract to the Central Brain Tumor Registry of the United States supported by the Pediatric Brain Tumor Foundation of the United States.

${ }^{2}$ Address correspondence and reprint requests to Bridget J. McCarthy, Division of Epidemiology and Biostatistics (M/C 922), School of Public Health, University of Illinois at Chicago, 2121 W. Taylor St., Chicago, IL 60612-7260.

${ }^{3}$ Abbreviations used are as follows: AAPC, average annual percentage change; CBTRUS, Central Brain Tumor Registry of the United States; ICDO, International Classification of Diseases of Oncology; NOS, not otherwise specified; PNET, primitive neuroectodermal tumor; SEER, Surveillance, Epidemiology and End Results. mas in the population aged 20 to 64 years, nerve sheath tumors in males, and pituitary tumors in females. Increases that were not specific to any population subgroup were seen for glioblastoma, oligodendrogliomas, and astrocytomas, excluding not otherwise specified (NOS) tumors. Corresponding decreases were noted for NOS, astrocytoma NOS, and glioma NOS. Increasing incidence trends for lymphomas were consistent with previous literature. Improvements in diagnostic technology in addition to changes in classification and coding were likely to be responsible for decreases seen in incidence of NOS subgroups and corresponding increases in glioma subgroups. In contrast, the increases identified for ependymomas, nerve sheath tumors, and pituitary tumors were less likely to be artifacts of improvements in diagnosis, and they warrant further study. Neuro-Oncology 3, 141-151, 2001 (Posted to Neuro-Oncology [serial online], Doc. 00-055, June 5, 2001. URL <neuro-oncology. mc.duke.edu>)

I nterest in brain tumor trends was sparked largely by reports in the late 1980s and early 1990s that mortality from cancer was increasing in industrialized nations (Bahemuka et al., 1988; Davis et al., 1988, 1991; La Vecchia and Decarli, 1985; Modan et al., 1992). Subsequent to these studies, brain tumor incidence was shown to have increased over time in children (Blair and Birch, 1994; Bunin et al., 1996; Gurney et al., 1996; Hjalmers et al., 1999; McKinney et al., 1994; Smith et al., 1998) as well as in the elderly (Ahsan et al., 1995; Greig et al., 1990; Legler et al., 1999; Mao et al., 1991; Polednak, 1991; Polednak and Flannery 1995; Werner et al., 1995) and has included both overall increases (Blair and Birch, 1994; Bunin et al., 1996; Greig et al., 1990; Gurney et al., 1996; Mao et al., 1991; McKinney et al., 1994; Polednak, 1991; Werner et al., 1995) as well as histologicspecific increases (Blair and Birch, 1994; Bunin et al., 
1996; Gurney et al., 1996; Hjalmers et al., 1999; McKinney et al., 1994; Werner et al., 1995). Incidence of brain lymphomas has increased due to the HIV/AIDS epidemic (Ahsan et al., 1995; Cote et al., 1996; Eby et al., 1988; Lutz and Coleman, 1994; Werner et al., 1995), but evidence indicates an increase in brain lymphomas in HIVnegative persons as well (Cote et al., 1996; Eby et al., 1988; Lutz and Coleman, 1994). This increase could affect overall brain tumor incidence trends when brain lymphomas are included in the definition of brain tumors.

An artifactual increase in brain tumor incidence has occurred due to improvements in diagnostic technology, most notably with the widespread use of CT and MRI scans beginning in the 1970s and 1980s (Desmeules et al., 1992; Legler et al., 1999; Mosso et al., 1992; Radhakrishnan et al., 1995; Steinberg, 1986; Steinberg et al., 1985). These new noninvasive diagnostic tools allowed clinicians to identify previously undiagnosed brain tumors. These changes may also have shifted a proportion of diagnoses from NOS $^{3}$ subgroups to more specific histologic subgroups; however, only one study of brain tumor incidence trends has specifically examined NOS tumors as a separate subgroup (Blair and Birch, 1994). In that study, incidence of NOS tumors decreased, but the magnitude of the decrease was not large enough to explain the whole increase in histologic-specific incidence. Whether the entire increase in brain tumor incidence can be attributed to changes in diagnostic technology is still debated (Desmeules et al., 1992; Forman, 1999; Legler et al., 1999; Smith et al., 1998; Varner, 1999).

During the early 1990s, revisions were made to both the World Health Organization's histologic classification scheme for CNS tumors (the "blue book"; Kleihues et al., 1993a, 1993b) and the ICDO (Percy et al., 1990). Included in these changes were the recognition of specific subtypes of ependymomas, and the tumor histology glioblastoma was moved from "Neuroepithelial tumors of uncertain origin" to "Astrocytic tumors" based on new scientific research (Kleihues et al., 1993a, 1993b). Although these changes are relatively recent, the authors of the blue book indicated that many of the additions represented histologies that were already being used in diagnosis over the decade prior to publication of the revision (Kleihues et al., 1993b). Several of the new entities in the blue-book revision did not have corresponding 4-digit histology codes in the ICDO (Davis et al., 1997; Kleihues et al., 1993a). A new version of the ICDO was published in 1990, and included a new scheme to code tumors by site (topography). As an example, version 1 has one site code for tumors occurring in the pituitary gland and the craniopharyngeal duct (194.3). Under the 1990 revision, these two sites are now coded separately (pituitary gland: C75.1; craniopharyngeal duct; C75.2). How this and other changes affected histologic-specific incidence rates is unclear, although registry data prior to 1990 was recoded to reflect the new scheme. Changes in brain tumor classification thus occurred for both neuropathologists (blue book) and for tumor registrars (ICDO), but the impact of these changes on reported incidence rates has not been documented.

Most reports on incidence trends of primary brain tumors have been limited to tumors of malignant behavior, as most population-based cancer registries only include reports on malignant tumors. Although this seems reasonable for most tumor sites, the clinical distinction of benign versus malignant is not as relevant for brain tumors because benign tumors located intracranially can have a poor prognosis similar to that of malignant tumors (Davis et al., 1996; Schoenberg et al., 1976). Further, restricting the reporting to malignant tumors has been shown to underestimate the total incidence of primary brain tumors by approximately $50 \%$ (Davis et al., 1997).

This report describes overall and histologic-specific incidence rate trends using a large population-based series of all primary brain and other CNS tumors from CBTRUS, a relatively new resource in which time trends have not been investigated (http://www.cbtrus.org). CBTRUS data are unique in that they include primary tumors of benign and uncertain behavior, thus allowing the exploration of trends for those histologic groups that contain both benign and malignant tumors, such as meningiomas and pituitary tumors (Davis et al., 1997; Surawicz et al., 1999).

\section{Methods}

\section{Data and Variable Definitions}

Data on all incident primary brain tumors diagnosed from 1985 through 1994 from six population-based state cancer registries were compiled as part of CBTRUS: the Connecticut Tumor Registry, Delaware State Tumor Registry, Cancer Data Registry of Idaho, Massachusetts Cancer Registry, Montana Central Tumor Registry, and Utah Cancer Registry. These states represent approximately $5 \%$ of the U.S. population. Primary tumors with the following ICDO site codes were included in the analysis (Percy et al., 1990): C70.0-C70.9 (meninges), C71.0-C71.9 (brain), C72.0-C72.9 (spinal cord, cranial nerves and other parts of the CNS), and C75.1-C75.3 (pituitary gland, craniopharyngeal duct, and pineal gland) and included tumors with behavior codes $\left(5^{\text {th }}\right.$ digit ICDO morphology) 0,1 , and 3 , which represent benign, uncertain, and malignant tumors, respectively. Available information included date of diagnosis, age at diagnosis, date of birth, sex, race, 5-digit ICDO morphology code (histology plus behavior), ICDO site code, and diagnostic confirmation.

Data were analyzed by major age groups, 0-19, 20-64, and $\geq 65$ years, and were analyzed separately by sex; one case in the original data coded as sex $=$ hermaphrodite was excluded. Cases were classified racially as White and Nonwhite. Tumors were considered microscopically confirmed (Cunningham et al., 1992) if diagnostic confirmation was coded as 1,2 , or 4 , which correspond to positive histology, positive exfoliative cytology, and microscopic confirmation with method not specified, respectively; codes $5,6,7,8$, and 9 (which correspond to positive lab test/marker, direct visualization, radiography and/or other imaging techniques, clinical diagnosis only, or unknown method of confirmation) were considered not confirmed. Tumors were divided into histologic subgroups developed by CBTRUS in conjunction with a neuropathologist 
and based on an integration of the WHO classification system and the ICDO histology coding system (Davis et al., 1996). The subgroups used with corresponding ICDO 4-digit histology codes (Percy et al., 1990) were as follows: astrocytoma, total (9383-9384, 9400-9424); Astrocytoma, NOS (9400); anaplastic astrocytoma (9401, 9411); astrocytoma, excluding NOS (9383-9384, 9401-9424); astrocytoma, excluding anaplastic (93839384, 9400, 9410, 9420-9424); pilocytic astrocytoma (9421); craniopharyngioma (9350); ependymoma, total (9391-9394); anaplastic ependymoma (9391-9393); glioblastoma (9440-9442); glioma, NOS (9380); Mixed glioma (9382); lymphoma (9590-9930); medulloblastoma/ PNET (9470-9473, 9501-9503); meningioma (9530-9534, 9537-9538); nerve sheath (9540-9560, 9570); NOS (8000-8009); oligodendroglioma, total (9450-9460); oligodendroglioma, excluding anaplastic (9450); pituitary (8140-8323); and all brain tumors and all brain tumors excluding lymphoma (which excludes histologies 9590-9930). For meningioma and nerve sheath tumors, one state was excluded from the analysis, as this state's registry indicated it does not collect data on nerve sheath tumors or spinal meningiomas (Surawicz et al., 1999).

Data quality was assessed with EDITS, a computer program developed by the Centers for Disease Control to edit cancer registry data (EDITS, 1997). The North American Association of Central Cancer Registries' callfor-data metafile from EDITS was modified by CBTRUS to perform edits on tumors of benign and uncertain behavior, in addition to malignant tumors. Errors were corrected prior to analysis. Additional information on the quality of CBTRUS data has been recently published (Surawicz et al., 2000).

Population data used for denominators in rates were obtained from the SEER program of the National Cancer Institute and made available by the U.S. Census Bureau (SEER, 1997). Data obtained were mid-year population estimates for each state by calendar year in 5-year age groups and stratified by race. Incidence rates were ageadjusted using the year 2000 population standard and the following age groups: 0-4, 5-9, 10-14, 15-19, 20-24, 25-29, 30-34, 35-39, 40-44, 45-49, 50-54, 55-59, 60-64, 65-69, 70-74, 75-79, 80-84, and 85+ years old (Anderson and Rosenberg, 1998).

\section{Statistical Analysis}

Descriptive information was tabulated for total numbers of tumors by age group, sex, race, tumor behavior, histology, and diagnostic confirmation. The proportion of microscopically confirmed tumors was tabulated for each year by state and age group.

Multiplicative Poisson regression was used to statistically compare trends over time (Esteve et al., 1994). Our strategy was to review overall trends first, then look at overall trends without lymphomas, to see if any increase persisted in the absence of lymphomas, which are known to be increasing. Then trends in histology groups were individually assessed, and factors that may influence rates by affecting classification (NOS categories) or diagnostic accuracy (microscopic confirmation) were evaluated. Models were fit using the proc genmod procedure in the SAS software system (SAS Software, 1993). The variable of interest was time, expressed as year of tumor diagnosis and coded as a continuous variable. Potential confounding effects were assessed for age group, sex, race, and microscopic confirmation. Trends were expressed as the AAPC over the 10-year period, with corresponding 2-sided $95 \%$ confidence intervals. Age group was coded as a categorical variable for the broad age groups $0-19,20-64$, and $\geq 65$ years old, and indicator variables were used for microscopic confirmation, sex, and race. Models were fit for total brain tumors and for the histologic subgroups. Hierarchical models with 2- and/or 3-way interaction terms for year with age group, sex, race and microscopic confirmation were assessed. When interaction terms were statistically significant, separate AAPCs were listed for the fully adjusted model (without interaction terms), as well as for the appropriate age, sex, or race strata (with the statistically significant interaction terms). Estimates for all strata relevant to the interaction term are reported in the tables (whether statistically significant or not) so the reader can review the consistency and direction of changes underlying each interaction. When data became too sparse for stratification and/or 3-way interaction, the highest level of interaction/stratification that gave informative results was reported. All final models were adjusted for age group, sex, race, and microscopic confirmation. Selected age-specific and ageadjusted estimates were graphed to illustrate trends in incidence over time. Incidence rates for the subgroups were not presented to focus the paper on changes over time rather than absolute values; incidence rates for CBTRUS data have been published elsewhere (Davis et al., 1996; Surawicz et al., 1999, 2000).

\section{Results}

\section{Description of Data}

The final data set included 16,078 primary brain tumors with over 1,400 tumors in each of the 10 years represented. About $9 \%$ of the tumors occurred in children (0-19 years old), whereas the majority (53\%) were in the 20 - to 64-year-old age group, with the remaining $38 \%$ occurring in the elderly ( $\geq 65$ years old). The data were evenly divided by sex ( $48 \%$ male, $52 \%$ female). Of note, most of the persons in the data set were white $(94 \%)$. About $45 \%$ of all tumors were nonmalignant $(5.0 \%$ uncertain, $39.9 \%$ benign).

Overall, $86 \%$ of the tumors had a microscopically confirmed diagnosis (Table 1). In the pediatric subgroup, microscopic confirmation was high, with $91 \%$ confirmation over the 10 -year period, and the yearly level ranging from $87 \%$ to $95 \%$. The highest levels of microscopic confirmation were seen in the age group 20 to 64 years old, where levels were $90 \%$ or greater for each year from 1985 through 1994 and 93\% overall. In the older age group ( $\geq 65$ years), the level of microscopic confirmation exceeded $80 \%$ only once in the 10 -year period and remained between $70 \%$ and $79 \%$ for the other years in the study with an overall level of $76 \%$, much lower than either the pediatric or young adult groups. Microscopic 
Table 1. Characteristics of the study population by histology

\begin{tabular}{|c|c|c|c|c|c|c|c|}
\hline \multirow[b]{2}{*}{ Histology } & \multirow[b]{2}{*}{ \% Histology confirmed } & \multicolumn{3}{|c|}{ Age at diagnosis (yrs) } & \multirow[b]{2}{*}{ Female (\%) } & \multirow[b]{2}{*}{ White (\%) } & \multirow[b]{2}{*}{ Total } \\
\hline & & $0-19$ & $20-64$ & $\geq 65$ & & & \\
\hline Glioblastoma & 92 & 49 & 1841 & 1866 & 44 & 97 & 3756 \\
\hline Astrocytoma & 96 & 563 & 1690 & 748 & 46 & 95 & 3001 \\
\hline Oligodendroglioma & 97 & 38 & 276 & 45 & 44 & 96 & 310 \\
\hline Medulloblastoma/PNET & 99 & 201 & 82 & 2 & 39 & 94 & 285 \\
\hline Ependymoma & 97 & 83 & 183 & 30 & 35 & 92 & 296 \\
\hline Mixed glioma & 100 & 26 & 124 & 11 & 40 & 94 & 161 \\
\hline Glioma NOS & 41 & 119 & 203 & 215 & 48 & 94 & 537 \\
\hline Meningioma & 85 & 25 & 1580 & 1696 & 71 & 92 & 3301 \\
\hline Nerve sheath & 96 & 21 & 511 & 134 & 54 & 93 & 666 \\
\hline Pituitary & 88 & 45 & 693 & 292 & 50 & 90 & 1030 \\
\hline Craniopharyngioma & 97 & 30 & 59 & 26 & 54 & 88 & 115 \\
\hline Lymphoma & 89 & 10 & 388 & 217 & 40 & 89 & 615 \\
\hline NOS tumors & 19 & 44 & 183 & 404 & 55 & 94 & 631 \\
\hline Total $^{\mathrm{a}}$ & 86 & 1484 & 8507 & 6087 & 52 & 94 & 16,078 \\
\hline
\end{tabular}

Study population was taken from population-based state cancer registries in Connecticut, Delaware, Idaho, Massachusetts, Montana, and Utah, for tumors diagnosed from 1985 through 1994.

${ }^{a}$ The sum of the subgroups listed in the table does not equal the total. Tumor types included in the total but not a specific subgroup include neoplasm, unspecified, benign and malignant neuronal/glial, neuronal and mixed, other mesenchymal, germ cell tumor, cysts, heterotopias, hemangiomas, hemangioblastoma, pineal parenchymal, chordoma/chondrosarcoma, choroid plexus, neuroepithelial, other tumors of cranial and spinal nerves.

confirmation also varied by tumor type with the lowest rates in the nonspecific NOS categories (Table 1).

Two collaborating states (Connecticut and Massachusetts) accounted for $68 \%$ of all cases reported. Each of the remaining regions (Delaware, Idaho, Montana, and Utah) accounted for less than $15 \%$ of cases. The percent of microscopically confirmed cases varied across regions, with $83 \%$ being the lowest and $87 \%$ the highest.

\section{Overall Incidence Trends}

Table 2A summarizes the results of Poisson regression analysis for all tumors and all tumors excluding brain lymphomas. Overall, the incidence of all primary brain tumors was modestly increasing. Males exhibited a statistically significant increased incidence over time for microscopically confirmed tumors, whereas females showed no significant increase. The incidence of nonmicroscopically confirmed tumors was decreasing, although this result was statistically significant only in females.

When brain lymphomas were excluded from the overall analysis, the increasing trend was diminished and no longer statistically significant. The sex differences in trend were no longer apparent. The effect of inclusion of lymphomas is illustrated in Fig. 1 for individuals 20 to 64 years old. Inclusion of the statistically significant interaction term (for microscopic confirmation by year) indicated that, for both sexes combined, the AAPC was increasing for microscopically confirmed tumors and decreasing for nonconfirmed tumors over this 10 -year period. The net effect was a small increase that was not statistically significant.

\section{Histologic-Specific Incidence Trends}

The incidence of brain lymphomas was increasing dramatically overall but concentrated in both the 20- to 64-year-old age group and males $\geq 65$ years (Table $2 \mathrm{~B}$; Fig. 1). No statistically significant trends were evident in females.

Trends for other histologic groups that displayed agespecific differences are listed in Table 3. A statistically significant increase in incidence of childhood (0-19 years) pilocytic astrocytomas occurred and was consistent by sex and race. No other histologies had statistically significant trends specific to the pediatric group.

Several histologic-specific trends were evident in adults (20-64 years old). The incidence of ependymomas, total and anaplastic, and all microscopically confirmed oligodendrogliomas were increasing at a statistically significant rate in this age group, as were anaplastic astrocytomas (Table 3). The incidence of astrocytomas, excluding anaplastic, was decreasing at a statistically significant rate.

Anaplastic astrocytomas were increasing about twice as fast in the elderly ( $\geq 65$ years) compared with those aged 20 to 64 years (Table 3). A decrease was seen in incidence of astrocytomas, excluding anaplastic, similar to the adult group. Other histologic subgroups did not have statistically significant trends specific to the elderly.

Two histologies, nerve sheath and pituitary tumors, had sex-specific trends. An AAPC of 5.9 was increasing at a statistically significant rate for incidence of nerve sheath tumors in males (Fig. 2) and a similar increase (AAPC of 5.9) was found for pituitary tumors in females (Fig. 2).

As indicated in Table 4 and Fig. 3A, categories of NOS tumors showed a decreasing trend over time. Similarly, the rate of tumors diagnosed without microscopic confirmation declined over time for glioblastoma and oligodendroglioma. Conversely, astrocytomas, excluding astrocytomas NOS, were statistically significantly increasing in incidence across all age, sex, and racial groups (Fig. 3B). Microscopically confirmed glioblastomas 
Table 2A. Overall incidence rate trends by AAPC for primary brain tumors, with and without lymphomas

\begin{tabular}{|c|c|c|c|}
\hline Subgroup & No. of cases & AAPC $(\%)$ & $95 \% \mathrm{CI}$ for AAPC \\
\hline All tumors ${ }^{a}$ & 16,078 & 0.9 & $0.4,1.4$ \\
\hline Males, microscopically confirmed ${ }^{\mathrm{a}}$ & 6807 & 1.9 & $1.1,2.7$ \\
\hline Males, not microscopically confirmed & 927 & -1.1 & $-2.6,0.5$ \\
\hline Females, microscopically confirmed & 7060 & 0.7 & $-0.1,1.5$ \\
\hline Females, not microscopically confirmed ${ }^{a}$ & 1284 & -2.2 & $-3.6,-0.7$ \\
\hline All tumors excluding lymphoma & 15,463 & 0.5 & $-0.1,1.1$ \\
\hline Microscopically confirmed ${ }^{a}$ & 13,321 & 1.0 & $0.4,1.6$ \\
\hline Not microscopically confirmed ${ }^{a}$ & 2142 & -2.5 & $-4.0,-1.1$ \\
\hline
\end{tabular}

For study population details, see Table 1. All models are Poisson regression and were adjusted for age group, sex, race, and microscopic confirmation. Subgroups are listed only when interaction terms with time had $P<0.05$. Subgroups are given when interaction terms with time had $P<0.05$.

${ }^{\mathrm{a}}$ The specific trend estimate is statistically significant.

Table 2B. Brain lymphoma incidence rate trends by AAPC

\begin{tabular}{|c|c|c|c|}
\hline Subgroup & No. of cases & $\operatorname{AAPC}(\%)$ & $95 \% \mathrm{Cl}$ for $\mathrm{AAPC}$ \\
\hline Lymphomas $^{\mathrm{a}}$ & 615 & 11.4 & $8.3,14.6$ \\
\hline \multicolumn{4}{|l|}{ Age 0 to $19 \mathrm{yrs}$} \\
\hline Microscopically confirmed ${ }^{\mathrm{b}}$ & 8 & -14.9 & $36.4,10.2$ \\
\hline Not microscopically confirmed ${ }^{b}$ & 2 & 64.5 & $-8.7,400.8$ \\
\hline \multicolumn{4}{|l|}{ Age 20 to 64 yrs } \\
\hline Males $^{\mathrm{a}}$ & 273 & 21.4 & $16.3,26.9$ \\
\hline Males, white, microscopically confirmed ${ }^{\mathrm{a}}$ & 197 & 16.2 & $10.4,22.4$ \\
\hline Males, white, not microscopically confirmed ${ }^{\mathrm{a}}$ & 40 & 37.5 & $21.9,57.2$ \\
\hline Males, nonwhite, microscopically confirmed ${ }^{\mathrm{a}}$ & 27 & 36.3 & $18.0,60.5$ \\
\hline Males, nonwhite, not microscopically confirmed ${ }^{\mathrm{a}}$ & 9 & 61.3 & $34.5,95.5$ \\
\hline Females, white, microscopically confirmed & 89 & 0.1 & $-6.9,7.6$ \\
\hline Females, white, not microscopically confirmed & 8 & -0.2 & $-19.8,180.8$ \\
\hline Females, nonwhite, microscopically confirmed & 16 & 14.1 & $-3.5,36.9$ \\
\hline Females, nonwhite, not microscopically confirmed & 2 & 13.8 & $-12.9,49.6$ \\
\hline \multicolumn{4}{|l|}{ Age $\geq 65$ years } \\
\hline Males $^{\mathrm{a}}$ & 90 & 14.1 & $8.0,20.7$ \\
\hline Males, white, microscopically confirmed ${ }^{\mathrm{a}}$ & 82 & 11.4 & $3.2,20.7$ \\
\hline Males, white, not microscopically confirmed & 4 & 28.4 & $-10.8,109.1$ \\
\hline Males, nonwhite ${ }^{b}$ & 4 & 20.7 & $-15.5,90.6$ \\
\hline Females, white, microscopically confirmed & 118 & 1.6 & $-4.5,8.3$ \\
\hline Females, white, not microscopically confirmed & 4 & 23.7 & $-13.5,95.5$ \\
\hline Females, nonwhite ${ }^{b}$ & 5 & 16.4 & $-15.0,70.3$ \\
\hline
\end{tabular}

For study population details, see Table 1. Poisson regression models are adjusted for age group, sex, race, and microscopic confirmation. $P$ values for statistically significant interaction terms are year $\times$ age, $P<0.01$; year $\times$ sex, $P<0.0000$; year $\times$ race, $P=0.0089$; year $\times$ microscopically confirmed, $P=0.0007$.

${ }^{a}$ The specific trend estimate is statistically significant.

${ }^{b}$ Stratified by age group and sex; sample size was insufficient to allow separate estimates by race for the population aged 0 to 19 years and by microscopic confirmation for $\geq 65$-yearold nonwhite males and females.

and oligodendrogliomas were also increasing, resulting in net increases (Table 4; Fig. 3B). Regression analysis indicated no increase or decrease in incidence for medulloblastoma/PNET, craniopharyngioma, meningioma, and mixed glioma (data not shown).

\section{Discussion}

With one exception (Radhakrishnan et al., 1995), this analysis represents the only U.S. study of brain tumor incidence trends to include all primary brain tumors, regardless of behavior. This inclusion allowed a more accurate analysis of trends in histologic subgroups that are of mixed behavior or are primarily benign in nature. Including all primary brain tumors from multiple regions also provided a sufficient number of tumors to examine trends in relatively rare histologic subgroups like oligodendrogliomas, ependymomas, and nerve sheath tumors. A separate analysis of NOS categories provided indirect evidence for a shift in the precision of diagnoses with the new diagnostic procedures.

Changes in overall brain tumor incidence trends due to increased use of CT and MRI in the 1970s and 1980s 


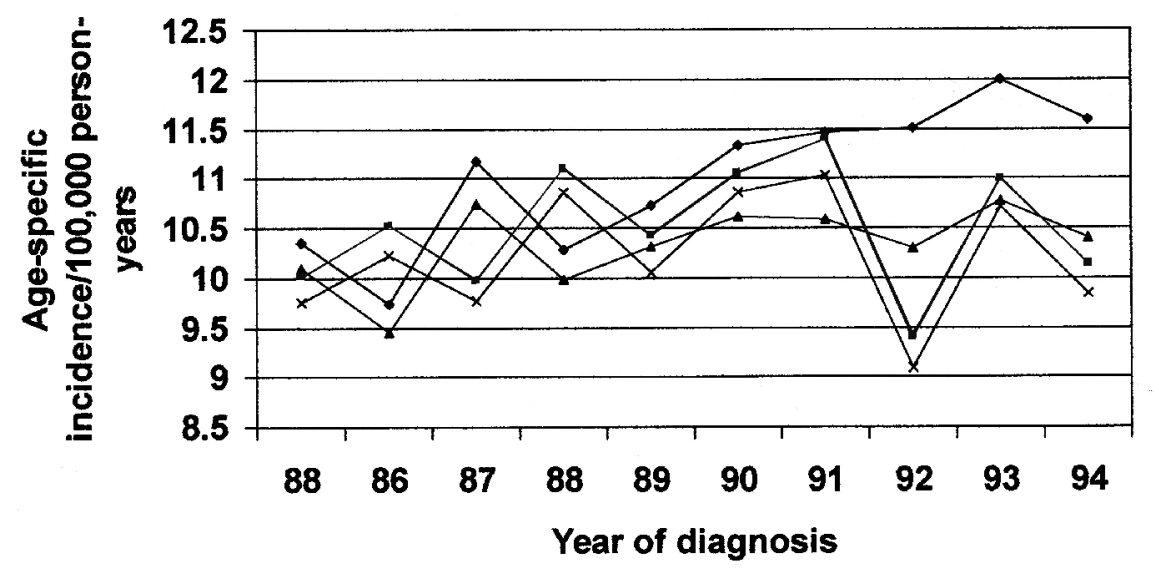

Fig. 1. Incidence of primary brain tumors in the 20- to 64-year-old age group. $\bullet$, Total males; $\mathbf{\square}$, total females; $\mathbf{\Lambda}$, males, excluding lymphomas; $X$, females, excluding lymphomas.

are fairly well established (Helseth, 1995; Legler et al., 1999; Modan et al., 1992; Radhakrishnan et al., 1995; Smith et al., 1998). If the increased use of these technologies was responsible for the observed increased incidence of brain tumors in the 1970s and 1980s, one would expect no increase or a very mild increase in brain tumor incidence from the time period reported here, 1985-1994. Indeed, when considered as a whole, excluding lymphomas, the incidence of primary brain tumors did not show a statistically significant increase; more pre-

Table 3. Tumor-specific incidence rate trends by AAPC with age-specific differences

\begin{tabular}{|c|c|c|c|}
\hline Histology by age & No. of cases & $\operatorname{AAPC}(\%)$ & $95 \% \mathrm{CI}$ for AAPC \\
\hline$\overline{\text { Ependymoma }^{a}}$ & 296 & 6.9 & $2.7,11.3$ \\
\hline $0-19$ yrs & 83 & 2.0 & $-5.4,9.9$ \\
\hline $20-64 \mathrm{yrs}^{\mathrm{a}}$ & 183 & 11.1 & $5.5,17.1$ \\
\hline$\geq 65$ yrs & 30 & -3.1 & $-14.6,9.8$ \\
\hline Anaplastic ependymoma ${ }^{a}$ & 253 & 7.0 & $2.4,11.7$ \\
\hline $0-19$ yrs & 75 & 0.9 & $-6.8,9.2$ \\
\hline $20-64 \mathrm{yrs}^{\mathrm{a}}$ & 149 & 12.2 & $5.9,19.0$ \\
\hline$\geq 65$ yrs & 29 & -1.7 & $-13.5,11.7$ \\
\hline Oligodendroglioma, total ${ }^{a}$ & 359 & 6.6 & $2.8,10.6$ \\
\hline $0-19^{b}$ yrs & 38 & -6.2 & $-16.3,4.8$ \\
\hline 20-64 yrs, microscopically confirmed ${ }^{\mathrm{a}}$ & 270 & 9.1 & $4.6,13.9$ \\
\hline $20-64$ yrs, not microscopically confirmed & 6 & -15.3 & $-34.3,5.9$ \\
\hline$\geq 65$ yrs, microscopically confirmed & 41 & 9.4 & $-1.3,21.6$ \\
\hline$\geq 65$ yrs, not microscopically confirmed & 4 & -15.1 & $-35.1,8.3$ \\
\hline Anaplastic astrocytoma $^{a}$ & 691 & 5.9 & $3.2,8.7$ \\
\hline $0-19$ yrs & 59 & -2.1 & $-10.5,6.9$ \\
\hline $20-64 \mathrm{yrs}^{\mathrm{a}}$ & 460 & 5.1 & $1.8,8.6$ \\
\hline$\geq 65$ yrs $^{\mathrm{a}}$ & 172 & 11.1 & $5.3,17.3$ \\
\hline Astrocytoma, excluding anaplastic ${ }^{a}$ & 2310 & -3.6 & $-4.9,-2.2$ \\
\hline $0-19$ yrs & 504 & 1.4 & $-1.6,4.5$ \\
\hline $20-64 \mathrm{yrs}^{\mathrm{a}}$ & 1230 & -5.5 & $-7.3,-3.6$ \\
\hline$\geq 65 \mathrm{yrs}^{\mathrm{a}}$ & 576 & -3.8 & $-6.5,-1.0$ \\
\hline Pilocytic astrocytoma ${ }^{a, c}$ & 163 & 13.8 & $7.7,20.4$ \\
\hline
\end{tabular}

For study population details, see Table 1. All models are Poisson regression and are adjusted for age group, sex, race, and microscopic confirmation. Subgroups are listed when interaction terms with time had $P<0.05$.

${ }^{a}$ The specific trend estimate is statistically significant.

${ }^{\mathrm{b}}$ The group aged 0 to 19 years could not be separated by microscopic confirmation due to insufficient cases of nonmicroscopically confirmed tumors.

cAnalysis of pilocytic astrocytomas was restricted to those aged 0 to 19 years. 


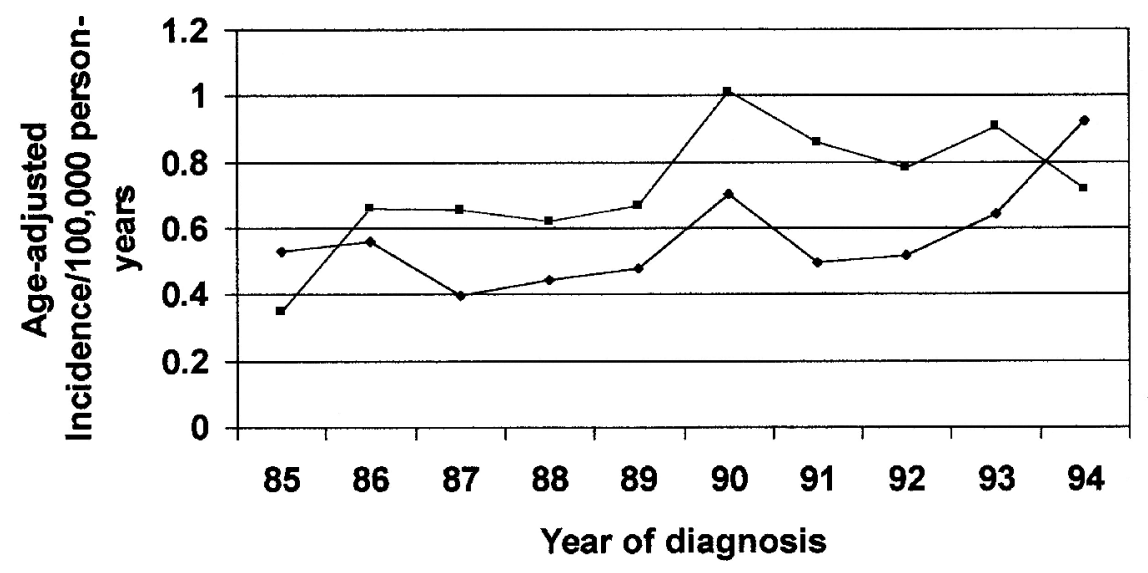

Fig. 2. Sex-specific incidence trends. $\bullet$, Nerve sheath (males); $\mathbf{\square}$, pituitary (females).

cisely, the incidence of microscopically confirmed tumors increased (AAPC 1.0\%), whereas the incidence of nonmicroscopically confirmed tumors decreased (AAPC $-2.5 \%$ ). This pattern suggests a further improvement in diagnosis, with no true increase in the occurrence of brain tumors during this time period. No changes in incidence were observed for the histologies medulloblastoma/PNET, craniopharyngioma, meningioma, and mixed glioma. However, other histologic-specific results do warrant further consideration.

Incidence of NOS tumors should be decreasing in most or all demographic strata if diagnostics and classification have improved. Indeed, several histologies with statistically significant decreases did not differ by sex, race, age group, or microscopic confirmation: NOS, astrocytomas NOS, and gliomas NOS. These three subgroups, which are mutually exclusive NOS categories, followed the expected pattern. Conversely, incidence rates in specified histologies should have increased over time. Although this was apparent, increases seen in glioblastoma, oligodendroglioma, and anaplastic astrocytoma were not limited to one population subgroup, suggesting multiple classification changes were occurring.

Overall, the incidence of astrocytomas decreased. Within this broad histologic group, though, statistically significant increases and decreases were detected. Ageadjusted incidence rates of anaplastic astrocytoma increased over the 10-year period, whereas astrocytomas, excluding anaplastic astrocytomas, decreased. Similarly, the incidence of astrocytomas NOS decreased, while astrocytomas, excluding NOS astrocytomas, increased. These results may be partially due to shifts

Table 4. Tumor-specific incidence rate trends by AAPC for histologies with nonspecific diagnosis or variation in trend by microscopic confirmation

\begin{tabular}{|c|c|c|c|}
\hline Histology & No. of cases & $\operatorname{AAPC}(\%)$ & $95 \% \mathrm{CI}$ for $\mathrm{AAPC}$ \\
\hline \multicolumn{4}{|c|}{ Categories with nonspecific diagnosis ${ }^{\mathrm{a}}$} \\
\hline $\mathrm{NOS}^{\mathrm{a}}$ & 631 & -8.6 & $-11.1,-6.0$ \\
\hline Glioma, NOS ${ }^{a}$ & 537 & -5.7 & $-8.5,-2.9$ \\
\hline Astrocytoma, total ${ }^{\mathrm{a}}$ & 3001 & -1.5 & $-2.7,-0.2$ \\
\hline Astrocytoma, NOS $^{a}$ & 1870 & -5.7 & $-7.2,-4.2$ \\
\hline Whites $^{\mathrm{a}}$ & 1787 & -5.3 & $-6.9,-3.8$ \\
\hline Nonwhites ${ }^{\mathrm{a}}$ & 83 & -12.7 & $-19.3,-5.8$ \\
\hline Astrocytoma, excluding $\operatorname{NOS}^{\mathrm{a}}$ & 1131 & 5.9 & $3.7,8.1$ \\
\hline \multicolumn{4}{|c|}{ Categories with changes in microscopic confirmation over time ${ }^{a}$} \\
\hline Glioblastoma $^{a}$ & 3756 & 2.3 & $1.1,3.4$ \\
\hline Microscopically confirmed ${ }^{a}$ & 3439 & 3.2 & $2.0,4.4$ \\
\hline Not microscopically confirmed ${ }^{a}$ & 317 & -7.2 & $-10.7,-3.5$ \\
\hline Oligodendroglioma & 310 & 3.8 & $-0.2,7.9$ \\
\hline Microscopically confirmed ${ }^{\mathrm{a}}$ & 303 & 4.4 & $0.4,8.7$ \\
\hline Not microscopically confirmed & 7 & -23.3 & $-45.6,1.3$ \\
\hline
\end{tabular}

For study population details, see Table 1. All models are Poisson regression and were adjusted for age group, sex, race, and microscopic confirmation. $P$ values for statistically significant interaction terms by subgroup are astrocytoma, NOS year $\times$ race, $P=0.0439$; glioblastoma, year $\times$ microscopically confirmed, $P<0.0000 ;$ oligodendroglioma, $P=0.0466$.

${ }^{a}$ The specific trend estimate is statistically significant. 
A

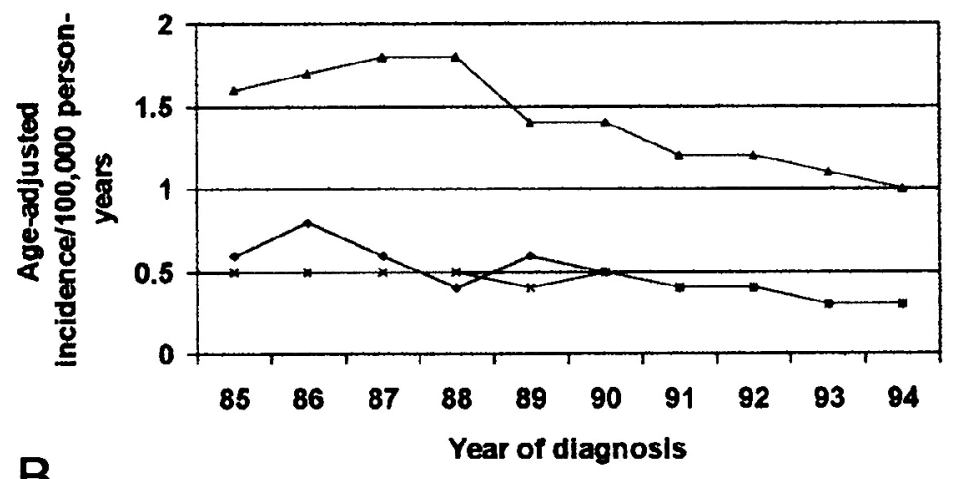

B

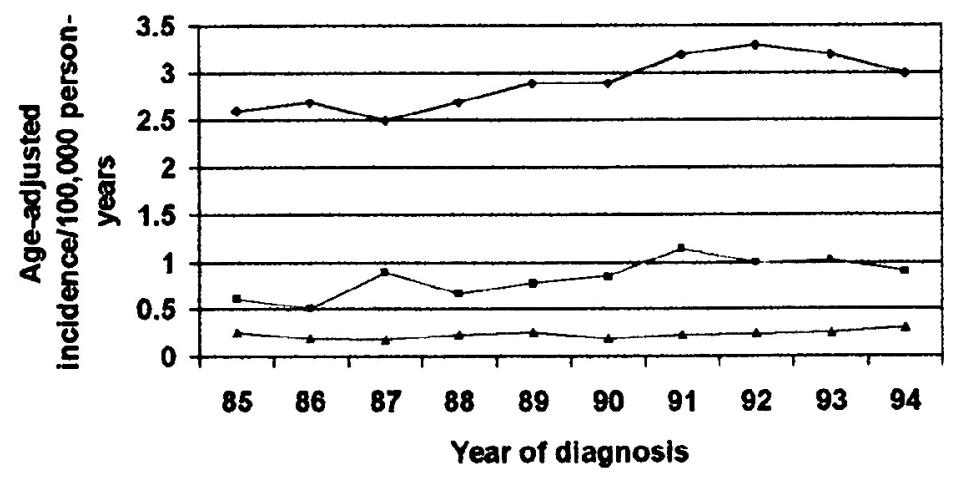

Fig. 3. Decreasing incidence trends in nonspecific categories (A) and corresponding increasing incidence trends in specific categories (B). A: $\bullet, N O S$; $\boldsymbol{\Lambda}$, astrocytoma, NOS; X, glioma, NOS. B: $\bullet$, Glioblastoma; $\boldsymbol{\square}$, astrocytoma, excluding NOS; $\boldsymbol{\Lambda}$, oligodendroglioma, microscopically confirmed.

toward more specific diagnoses within the broad astrocytoma group, for instance, from astrocytomas NOS to anaplastic astrocytomas. Because all of these groups are not mutually exclusive (astrocytomas excluding anaplastic includes the astrocytomas NOS, and anaplastic astrocytomas are included in astrocytomas excluding NOS) the findings are likely to be explained by the same shifts in specificity.

The results for astrocytomas highlight the need for good quality data. It is disconcerting that the largest histology within the astrocytoma group was astrocytomas NOS (62\% of all astrocytomas in the data), which is the least specific astrocytoma histology. The recent revision of the blue book has included a new scheme for histologic classification of astrocytomas based on grade (Kleihues et al., 1993a, 1993b). It is hoped that standardizing these classifications along with further molecular diagnostic improvements will lead to a clarification in classification and reporting of astrocytomas, reducing the proportion of unspecified astrocytoma tumor types.

Increases in incidence of total oligodendrogliomas were statistically significant only in individuals aged 20 to 64 years. This trend also may be driven by classification changes. First, oligodendrogliomas belong to the larger group of gliomas that includes glioblastomas and astrocytomas, the other subgroups likely to have absorbed the shift from unspecified tumors. The age group specificity disappeared when anaplastics were removed, but anaplastic oligodendrogliomas were too rare to be analyzed separately. The pattern of incidence rates for both oligodendroglial groups increased for microscopically confirmed tumors and decreased for nonmicroscopically confirmed tumors. Second, the increase in incidence of oligodendrogliomas may be the result of neuropathologists' "looking harder" for a tumor now known to be chemosensitive and amenable to treatment in up to $70 \%$ of anaplastic and aggressive oligodendroglioma cases (Cairncross et al., 1992; Paleologos et al., 1999). A shift from unspecified to a more specific tumor classification may be occurring in an effort to improve diagnosis and, thus, treatment of these tumors.

The incidence of pituitary tumors increased in white females (AAPC $5.9 \%$ ). An increase of pituitary tumors was also noted in Rochester, Minn., over 4 decades (Radhakrishnan et al., 1995), but sex and/or race differences were not reported in that analysis. Increases in incidence of pituitary tumors were the only histologic subgroup in the Rochester study that could not be explained by temporal changes in microscopic confirmation. This may be a real rather than an artifactual increase.

The incidence of nerve sheath tumors increased almost $6 \%$ per year in males. The etiology of these primarily 
benign tumors is unknown. Exposure to electromagnetic fields, such as cellular telephone use, has been implicated in the development of nerve sheath tumors and a prospective study on associations between mobile phone use and brain tumors (as well as other head and neck cancers) is underway (Anonymous, 1998). Occupational exposure to noise was associated with increased risk for development of acoustic schwannomas in men (Preston-Martin et al., 1989); women were not included in the analysis. In light of these results, it is interesting that the increase in nerve sheath tumors in this analysis is specific to males.

The incidence of brain lymphoma increased in both the adult (20-64 years) and elderly ( $\geq 65$ years) age groups. The increases were statistically significant in 20- to 64-year-old males within all race and microscopic confirmation groups and in white males 65 years or older. This finding corroborates reported results from several studies (Ahsan et al., 1995; Cote et al., 1996; Eby et al., 1988; Lutz and Coleman, 1994; Werner et al., 1995). Much, but not all of the increase, may be attributed to the AIDS epidemic (Cote et al., 1996; Eby et al., 1988; Lutz and Coleman, 1994).

The increased incidence of ependymomas (both total and anaplastic) was confined to adults (20-64 years) and contributed to overall increases in the incidence of ependymoma for all ages combined. Similar findings have not been reported in other studies. The incidence of ependymomas peaks between 35 and 55 years old (Surawicz et al., 1999), suggesting this trend in the adult population could be of etiologic importance.

This study included analysis of histologic subgroups that are most common in the pediatric population: medulloblastomas/PNETs, ependymomas, craniopharyngiomas, and pilocytic astrocytomas. No changes in the incidence of childhood medulloblastomas/PNETs were evident, in contrast to several earlier reports of increased incidence (Blair and Birch, 1994; Bunin et al., 1996; McKinney et al., 1994). Pilocytic astrocytoma was the only childhood tumor that had a statistically significant increase during the study period. It is likely that a proportion of the increase in pilocytic astrocytomas can be explained by decreases in the incidence of astrocytomas NOS in this age group, the first and third most common pediatric brain tumors in CBTRUS data, respectively (Surawicz et al., 1999). If this is the case, then these results are also in contrast to earlier reports of increases in incidence of astroglial tumors (Blair and Birch, 1994; Gurney et al., 1996); however, these early studies did not examine trends for NOS subgroups separately.

In their recent analysis of SEER data, Smith et al. (1998) demonstrated that the increase in childhood brain tumor incidence was best explained with a "jump" in incidence that occurred around 1984-1985, rather than a constant rate of increase. According to their analysis, incidence rates for pediatric brain tumors increased sharply in the mid 1980s (presumably due to a surge in MRI use) and have remained stable since that time. With respect to the pediatric tumors, our results are consistent with that study. Our data begin in 1985 after these major diagnostic changes took place, so a direct assessment of this phenomenon was not possible.
If improvements in diagnostics and tumor classification were the sole explanation for brain tumor incidence trends, one would expect trends to be most evident in the elderly, where most of the diagnostic change has occurred in the United States (Gibson et al., 1984; Legler et al., 1999). Several studies have reported incidence increases in the elderly (Greig et al., 1990; Legler et al., 1999; Mao et al., 1991; Polednak, 1991; Werner et al., 1995). A recent study by Legler et al. (1999) reported increased incidence in the elderly with no increase in younger adults over the last 2 decades. That study also gave evidence that rates of CT, MRI, and stereotactic biopsy continued to rise during the period 1986-1994. However, in the present study, which also encompasses these dates, no increases in the elderly were detected. If, alternatively, improved diagnostics and classification were equal in all segments of the population, histologic subgroups should not exhibit trends specific to different demographic strata such as race or sex. This was true for histologic subgroups such as astrocytomas, glioblastomas, and oligodendrogliomas in this study; however, as discussed above, unexplained sex-specific trends were also identified for three histologies. Taken together, the results obtained in this study do not support diagnostic changes as the full explanation for changes in incidence over the last decade.

Ten years of data may be insufficient to accurately describe true changes in incidence over time, yet statistically significant trends (both increasing and decreasing) were identified in these data. Having such recent data did not allow incidence trends to be analyzed in terms of the full spectrum of diagnostic changes that have occurred over the last 25 to 30 years; however, this caveat served to eliminate major changes in incidence caused by CT scanning, and probably greatly reduced changes in incidence brought on by use of MRI. Because the method used to diagnose tumors initially is not included in the available registry data, lingering effects of these changes could not be fully explored.

CBTRUS is a relatively new data resource, and differences by region have not been fully explored. A recent study of CBTRUS data indicated differences in incidence by region, likely due to differences in reporting practices (Surawicz et al., 2000). Differences in pathologic diagnosis by state have also been suggested (Velema and Percy, 1987). Efforts to assess regional differences in incidence rates await further standardization of diagnosis and reporting. As an attempt to control for differences in reporting practices by region, microscopic confirmation was controlled for in all analyses. Microscopic confirmation varied from $83 \%$ to $87 \%$ between states for all years combined, and from $77 \%$ to $94 \%$ when each year was considered separately (data not shown). Also, based on the populations of the included states, the results largely reflect patterns for the northeastern United States and are most informative for whites compared with other race/ethnic groups, whose numbers were often too small for meaningful analysis.

In spite of these possible limitations, this analysis contributes important information to the debate over brain tumor incidence rate trends. Most analyses in the United States have used SEER data, especially in adults. 
Although SEER and CBTRUS are not mutually exclusive (two regions overlap), trend analyses in different populations are necessary to further separate artifactual patterns from potential true changes. Further, CBTRUS includes information on all primary brain tumors, regardless of behavior. Because several brain tumor subtypes may have benign and malignant entities, or progress from benign to malignant, this inclusion provided a more complete description of trends.

This analysis documented no overall change in incidence of primary brain tumors and confirmed the increase in brain lymphomas. Incidence decreased in NOS histologic subgroups and increased in specific glioma subtypes; this finding supported previous observations that diagnostic methods and tumor classification over the period 1985-1994 have improved. Diagnostic improvements seem unlikely to be responsible for the entire pattern of trends for pituitary tumors, nerve

sheath tumors, and ependymomas. These patterns warrant further study.

\section{Acknowledgments}

The authors wish to gratefully acknowledge the Central Brain Tumor Registry of the United States and the collaborating registries that provided data for this analysis:

\begin{tabular}{|c|c|}
\hline Dr. Anthony Polednak & Connecticut Tumor Registry \\
\hline Ms. Carol Marker & $\begin{array}{l}\text { Delaware State Tumor } \\
\text { Registry }\end{array}$ \\
\hline Ms. Stacey Carson & Cancer Data Registry of Idaho \\
\hline Dr. Susan Gershman & Massachusetts Cancer Registry \\
\hline Ms. Debbi Lemons & $\begin{array}{l}\text { Montana Central Tumor } \\
\text { Registry }\end{array}$ \\
\hline Ms. Rosemary Dibble & Utah Cancer Registry \\
\hline
\end{tabular}

Dr. Anthony Polednak

\author{
Connecticut Tumor Registry \\ Delaware State Tumor \\ Registry \\ Montana Central Tumor \\ Utah Cancer Registry
}

\section{References}

Ahsan, H., Neugut, A.I., and Bruce, J.N. (1995) Trends in incidence of primary malignant brain tumors in USA, 1981-1990. Int. J. Epidemiol. 24, 10781085.

Anderson, R.N., and Rosenberg, H.M. (1998) Age standardization of death rates: Implementation of the year 2000 standard. Natl. Vital Stat. Rep. 47,1-16, 20.

Anonymous (1998) Of mobile phones and morbidity. Environ. Health Perspect. 106, A474-A475.

Bahemuka, M., Massey, E.W., and Schoenberg, B.S. (1988) International mortality from primary nervous system neoplasms: Distribution and trends. Int. J. Epidemiol. 17, 33-38.

Blair, V., and Birch, J.M. (1994) Patterns and temporal trends in the incidence of malignant disease in children. II. Solid tumours of childhood. Eur. J. Cancer 30A, 1498-1511.

Bunin, G.R., Feuer, E.J., Witman, P.A., and Meadows, A.T. (1996) Increasing incidence of childhood cancer: Report of 20 years experience from the Greater Delaware Valley Pediatric Tumor Registry. Pediatr. Perinat. Epidemiol. 10, 319-338.

Cairncross, J.G., Macdonald, D.R., and Ramsay, D.A. (1992) Aggressive oligodendroglioma: A chemosensitive tumor. Neurosurgery 31, 78-82.

Cote, T.R., Manns, A., Hardy, C.R., Yellin, F.J., and Hartge, P. (1996) Epidemiology of brain lymphoma among people with or without acquired immunodeficiency syndrome. AIDS/Cancer Study Group. J. Natl. Cancer Inst. 88, 675-679.

Cunningham, J., Ries, L., Hankey, B., Seiffert, J., Lyles, B., Shambaugh, E., Percy, C., and Van Holten, V. (1992) The SEER Program Code Manual. Revised Edition. Bethesda, Md: National Cancer Institute, National Institutes of Health.

Davis, D.L., and Schwartz, J. (1988) Trends in cancer mortality: US white males and females, 1968-83. Lancet. 1, 633-636.

Davis, D.L., Ahlbom, A., Hoel, D., and Percy, C. (1991) Is brain cancer mortality increasing in industrialized countries? Am. J. Ind. Med. 19, 421-431.

Davis, F.G., Malinski, N., Haenszel, W., Chang, J., Flannery, J., Gershman, S., Dibble, R., and Bigner, D.D. (1996) Primary brain tumor incidence rates in four United States regions, 1985-1989: A pilot study. Neuroepidemiology 15, 103-112.

Davis, F.G., Bruner, J.M., and Surawicz, T.S. (1997) The rationale for standardized registration and reporting of brain and central nervous system tumors in population-based cancer registries. Neuroepidemiology 16 , 308-316.
Desmeules, M., Mikkelsen, T., and Mao, Y. (1992) Increasing incidence of primary malignant brain tumors: Influence of diagnostic methods. J. Natl. Cancer Inst. 84, 442-445.

Eby, N.L., Grufferman, S., Flannelly, C.M., Schold, S.C., Jr., Vogel, F.S., and Burger, P.C. (1988) Increasing incidence of primary brain lymphoma in the US. Cancer 62, 2461-2465.

EDITS [computer program] (1997) Version 2.00. Atlanta, Ga: Division of Cancer Prevention and Control, National Center for Chronic Disease Prevention and Health Promotion, Centers for Disease Control and Prevention.

Esteve, J., Benhamou, E., and Raymond, L. (1994) Statistical Methods in Cancer Research. Volume IV. Descriptive Epidemiology. Lyon: International Agency for Research on Cancer.

Forman, A.D. (1999) Re: Trends in reported incidence of primary malignant brain tumors in children in the United States. J. Natl. Cancer Inst. 91, 648-649.

Gibson, R.M., Levit, K.R., Lazenby, H., and Waldo, D.R. (1984) National health expenditures, 1983. Health Care Financing Rev. 6, 1-29.

Greig, N.G., Ries, L.G., Yancik, R., and Rapoport, S.I. (1990) Increasing annual incidence of primary malignant brain tumors in the elderly. J. Natl. Cancer Inst. 82, 1621-1624.

Gurney, J.G., Davis, S., Severson, R.K., Fang, J.Y., Ross, J.A., and Robison, L.L. (1996) Trends in cancer incidence among children in the US. Cancer 78, 532-541.

Helseth, A. (1995) The incidence of primary central nervous system neoplasms before and after computerized tomography availability. J. Neurosurg. 83, 999-1003.

Hjalmers, U., Kulldorff, M., Wahlqvist, Y., and Lannering, B. (1999) Increased incidence rates but no space-time clustering of childhood astrocytoma in Sweden, 1973-1992. Cancer 85, 2077-2090.

Kleihues, P., Burger, P.C., and Scheithauer, B.W. (1993a) Histological Typing of Tumours of the Central Nervous System. Second edition. Berlin: SpringerVerlag.

Kleihues, P., Burger, P.C., and Scheithauer, B.W. (1993b) The new WHO classification of brain tumors. Brain Pathol. 3, 255-268.

La Vecchia, C., and Decarli, A. (1985) Trends in cancer mortality in Italy, 19551978. Tumori 71, 201-218.

Legler, J.M., Gloeckler Ries, L.A., Smith, M.A., Warren, J.L., Heineman, E.F., Kaplan, R.S., and Linet, M.S. (1999) Brain and other central nervous system cancers: Recent trends in incidence and mortality. J. Natl. Cancer Inst. 91, 1382-1390. 
Lutz, J.M., and Coleman, M.P. (1994) Trends in primary cerebral lymphoma. Br. J. Cancer 70, 716-718.

Mao, Y., Desmeules, M., Semenciw, R.M., Hill, G., Gaudette, L., and Wigle, D.T. (1991) Increasing brain cancer rates in Canada. Can. Med. Assoc. J. 145, 1583-1591.

McKinney, P.A., Ironside, J.W., Harkness, E.F., Arango, J.C., Doyle, D., and Black, R.J. (1994) Registration quality and descriptive epidemiology of childhood brain tumours in Scotland 1975-90. Br. J. Cancer 70, 973-979.

Modan, B., Wagener, D.K., Feldman, J.J., Rosenberg, H.M., and Feinleib M. (1992) Increased mortality from brain tumors: A combined outcome of diagnostic technology and change of attitude toward the elderly. Am. J. Epidemiol. 135, 1349-1357.

Mosso, M.L., Colombo, R., Giordano, L., Pastore, G., Terracini, B., and Magnani, C. (1992) Childhood Cancer Registry of the province of Torino, Italy: Survival, incidence, and mortality over 20 years. Cancer 69, 1300-1306.

Paleologos, N.A., Macdonald, D.R., Vick, N.A., and Cairncross, J.G. (1999) Neoadjuvant procarbazine, CCNU, and vincristine for anaplastic and aggressive oligodendroglioma. Neurology 53, 1141-1143.

Percy, C., Van Holten, V., and Muir, C.S. (Eds.) (1990) International Classification of Diseases for Oncology. Second edition. Geneva: World Health Organization.

Polednak, A.P. (1991) Time trends in incidence of brain and central nervous system cancers in Connecticut. J. Natl. Cancer Inst. 83, 1679-1681.

Polednak, A.P., and Flannery, J.T. (1995) Brain, other central nervous system, and eye cancer. Cancer 75, 330-337.

Preston-Martin, S., Thomas, D.C., Wright, W.E., and Henderson, B.E. (1989) Noise trauma in the aetiology of acoustic neuromas in men in Los Angeles County, 1978-1985. Br. J. Cancer 59, 783-786.

Radhakrishnan, K., Mokri, B., Parisi, J.E., O'Fallon, W.M., Sunku, J., and Kurland, L.T. (1995) The trends in incidence of primary brain tumors in the population of Rochester, Minnesota. Ann. Neurol. 37, 67-73.

SAS Technical Report P-243, SAS/STAT Software: The GENMOD Procedure, Release 6.09. (1993) Cary, N.C.: SAS Institute.
Schoenberg, B.S., Christine, B.W., and Whisnat, J.P. (1976) The descriptive epidemiology of primary intracranial neoplasms: The Connecticut experience. Am. J. Epidemiol. 104, 499-510.

Smith, M.A., Freidlin, B., Gloeckler Ries, L.A., and Simon, R. (1998) Trends in reported incidence of primary malignant brain tumors in children in the United States. J. Natl. Cancer Inst. 90, 1269-1277.

Steinberg, E.P. (1986) The status of MRI in 1986: Rates of adoption in the United States and elsewhere. Am. J. Roentgenol. 147, 435-455.

Steinberg, E.P., Sisk, J.E., and Locke, K.E. (1985) X-ray CT and magnetic resonance imagers: Diffusion patterns and policy issues. N. Engl. J. Med. 313, 859-864.

Surawicz, T.S., McCarthy, B.J., Kupelian, V., Jukich, P.J., Bruner, J.M., Davis, F.G., and the collaborating registries of the Central Brain Tumor Registry of the United States (1999) The descriptive epidemiology of primary brain and CNS tumors: Results from the Central Brain Tumor Registry of the United States, 1990-1994. Neuro-Oncology [serial on line], Doc. \#98-13, January 19, 1999. URL neuro-oncology.mc.duke.edu. Neuro-Oncol. 1, 14-25.

Surawicz, T.S., McCarthy, B.J., Jukich, P.J., and Davis, F.G. (2000) The accuracy and completeness of primary brain and central nervous system tumor data: Results from the Central Brain Tumor Registry of the United States. J. Registry Manag. 27, 51-55.

Surveillance, Epidemiology, and End Results (SEER) (1997) U.S. Populations, 1973-1996. Bethesda, Md.: National Cancer Institute, November 11, 1997. URL www.seer.ims.nci.nih.gov.

Varner, A. (1999) Re: Trends in reported incidence of primary malignant brain tumors in children in the United States. J. Natl. Cancer Inst. 91, 973-974.

Velema, J.P., and Percy, C.L. (1987) Age curves of central nervous system tumor incidence in adults: Variation of shape by histologic type. J. Natl. Cancer Inst. 79, 623-629.

Werner, M.H., Phuphanich, S., and Lyman, G.H. (1995) The increasing incidence of malignant gliomas and primary central nervous system lymphoma in the elderly. Cancer 76, 1634-1642. 